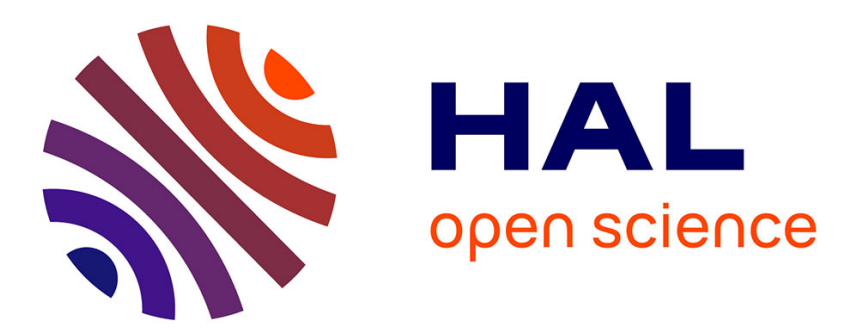

\title{
A finite element approach of the behaviour of woven materials at microscopic scale
}

\author{
Damien Durville
}

\section{To cite this version:}

Damien Durville. A finite element approach of the behaviour of woven materials at microscopic scale. 11th Euromech-Mecamat conference - Mechanics of microstructured solids : cellular materials, fiber reinforced solids and soft tissues, Mar 2008, Torino, Italy. hal-00274058

\section{HAL Id: hal-00274058 \\ https://hal.science/hal-00274058}

Submitted on 17 Apr 2008

HAL is a multi-disciplinary open access archive for the deposit and dissemination of scientific research documents, whether they are published or not. The documents may come from teaching and research institutions in France or abroad, or from public or private research centers.
L'archive ouverte pluridisciplinaire HAL, est destinée au dépôt et à la diffusion de documents scientifiques de niveau recherche, publiés ou non, émanant des établissements d'enseignement et de recherche français ou étrangers, des laboratoires publics ou privés. 


\title{
A finite element approach of the behaviour of woven materials at microscopic scale
}

\author{
Damien Durville
}

\begin{abstract}
A finite element simulation of the mechanical behaviour of woven textile materials at the scale of individual fibers is proposed in this paper. The aim of the simulation is to understand and identify phenomena involved at different scales in such materials. The approach considers small patches of woven textile materials as collections of fibers. Fibers are modelled by $3 \mathrm{D}$ beam elements, and contact-friction interactions are considered between them. An original method for the detection of contacts, and the use of efficient algorithms to solve the nonlinearities of the problem, allow to handle patches made of few hundreds of fibers. The computation of the unknown initial configuration of the woven structure is carried out by simulating the weaving process. Various loading cases can then be applied to the studied patches to identify their mechanical characteristics. To approach the mesoscopic behaviour of yarns, 3D strains are calculated at the scale of yarns, as post-processing. These strains display strong inhomogeinities, which raises the question of using continuous models at the scale of yarns.
\end{abstract}

\section{Introduction}

The mechanics of woven materials can be adressed at three different scales : the macroscopic scale relevant to pieces of fabric, the mesoscopic scale related to yarns, and the microscopic scale concerning fibers inside yarns. Different approaches are available to handle the problem of the identification and characterization of the complex mechanical behaviour of these materials. Some developments are specially dedicated to the characterization of the geometry of yarns in the woven structure (酒, 5). Geometries of yarns obtained by these methods can then be meshed and classical finite element codes can be employed to compute the mechanical response of textile

Damien Durville

LMSSMat - Ecole Centrale Paris. Grande Voie des Vignes, 92290 Chatenay-Malabry, France, e-mail: damien.durvilledecp.fr 
or textile composite structures. Other approaches concentrate on the finite element simulation at the scale of yarns, representing yarns by means of 3D elements with appropriate constitutive laws [1]. Going down to the scale of fibers requires a description of the geometry of fibers in the initial configuration. Such a geometry is a priori unknown, and therefore needs to be computed, for example by simulating the weaving process. Such an approach has been proposed using a finite element simulation code based on an explicit solver [đ]. The method we present here is based on a simulation code specially developped to handle the mechanical behaviour of entagled media [2], and makes use of an implicit solver. This simulation code is able to handle small patches of fabric made of few hundreds of fibers in order to identify their mechanical behaviour [3]. After recalling some basic features of the method, the way the weaving process is simulated to compute the initial configuration of the woven structure is presented, and results for two different weave patterns are given. Classical loading tests are then applied to these patches. The last part is dedicated to the calculation of 3D strains at the scale of yarns by means of meshes generated as post-processing. The results show strong inhomegeneities of strains at the scale of yarns, induced by localized displacements taking place between fibers.

\section{Purposes of the simulation at microcopic scale}

The needs related to the modelling and simulation of textile materials are diversified. Formability studies require an identification of the global macroscopic behaviour of fabrics, whereas the focus will be put on the local behaviour of individual fibers if damage and fiber breaking phenomena are to be investigated.

The coupling between phenomena at different scales makes the study of such materials complex. The two main mechanisms ruling the behaviour of textile materials, namely the interweaving of yarns constituting the fabric, and the entanglement of fibers within the yarns, occur at different scales but must be considered simultaneaously. One possible strategy to solve the problem at the macroscopic scale is to formulate a mesoscopic model for yarns accounting for the behaviour of the bundles of fibers making up the yarns. However, at the scale of yarns, one important issue is to determine whether phenomena between discrete fibers within yarns may be approached by continuous models.

To explore these questions, we propose to adress the global problem at the microscopic scale of individual fibers, by considering small patches of fabrics as assemblies of individual fibers, arranged in bundles, and developping between them contact-friction interactions. By this way, models at intermediate scales are no longer required, and the only mechanical characteristics to be known are those of individual fibers. As a downside, the initial geometry of fibers in the woven structure can not be predicted a priori. It is therefore necessary to compute this initial geometry by simulating the weaving process. 


\section{Main features of the simulation code}

\subsection{A kinematically enriched beam model for fibers}

The beam elements used to take into account fibers in the simulation are based on an enriched kinematical model that describes kinematics of each cross-section by the means of three vector fields : one for the translation of the centroid, and two to represent planar and linear deformations of the cross-section. This model, characterized by nine degrees of freedom, allows to calculate full 3D strain tensors, accounting for deformations of cross-sections.

\subsection{Modelling of contact between fibers}

An original method [2, 3] has been developped to detect quicky and accurately the numerous contacts taking place within a collection of fibers submitted to large deformations. This method is based on the determination of contact elements made of pairs of material particles that are predicted to enter into contact. The determination of these elements relies on the construction of intermediate geometries in any region where two parts of beam are sufficiently close to each other. These geometries are defined as the average of the two close parts of line. Normal directions to these geometries are used as contact search directions to define material particles of contact elements. By this way, the process of determination of contact elements is symmetrical with respect to the two considered beams - which is not the case when the normal direction to only one structure is chosen as contact search direction.

\subsection{Rigid bodies for the driving of boundary conditions}

To simulate the various conditions corresponding to the initial weaving process or the different loading tests, a versatile driving of boundary considitions is required. For this purpose, rigid bodies are attached to each end of yarn, and to each side of the patch. These rigid bodies can be driven either by displacements or by forces, applied to their degrees of freedom in both translation and rotation. 


\section{Computation of the initial configuration : simulation of the weaving process}

\subsection{Modelling of the weaving process}

The simulation of the weaving process is necessary to determine the unknown initial configuration of the woven structure. The weave pattern specifies which yarn must above or below the other at each crossing. The basic idea to simulate the weaving process is to make these conditions progressively fulfiled.

To do so, we start from a flat configuration where all yarns are straight and penetrate each other at crossings. Then, for few steps, for any penetration detected between two fibers belonging to crossing yarns, we take as normal direction of contact a vertical direction oriented according to the local crossing order prescribed by the weave pattern. This process is applied until yarns are completely separated at crossings. Then, in a second stage, classical contact directions, depending only on the local geometry of fibers, are considered, while forces and displacements applied on the sides of the patch are progressively relaxed. At the end of this process, an equilibrium configuration of the woven structure after weaving is obtained.

\subsection{Application to the studied patches}

Two patches of fabric, made with the same yarns, but according to two different weave patterns -a plain weave and a twill weave-, have been considered for the results presented here. The main features characterizing these patches are given in Table 11. Each patch is made of 408 fibers, and about 80.000 contact elements are considered in the simulation.

\begin{tabular}{lr}
\hline Nb. of fibers weft yarns & 44 \\
Nb. of fibers warp yarns & 24 \\
Nb. of weft yarns & 6 \\
Nb. of warp yarns & 6 \\
Total nb. of fibers & 408 \\
Nb. of nodes & 35.000 \\
Nb. of dofs & 300.000 \\
Nb. of contact elements & $\approx 80.000$ \\
\hline
\end{tabular}

Table 1 Characteristics of the studied patches

Computed configurations for the two weavings are shown on Fig. 4.2. Cuts of the two computed initial configurations and details of these cuts (Fig. 21) show the rearrangement of fibers and typical shapes of cross-sections obtained by the simulation. Whereas for plain weaves shapes of yarn cross-sections are predominantly 
lenticular, for twill weave cross-sections are more complex and vary along the yarn (Fig. 3).

(b)

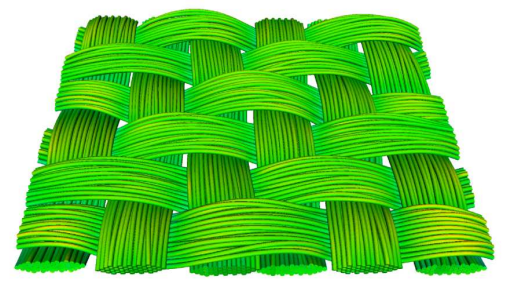

(c)

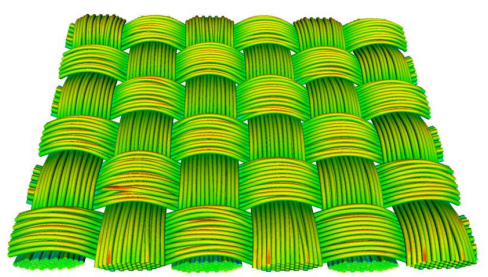

Fig. 1 Simulation of the weaving process : configuration before weaving (a), computed configuration for plain weave (b) and twill weave (c)

(a)

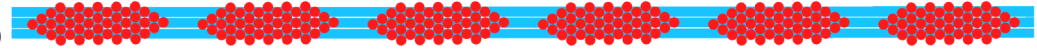

(b)

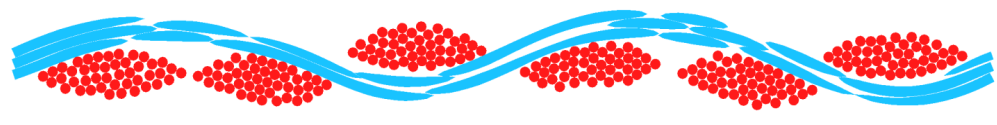

(c)

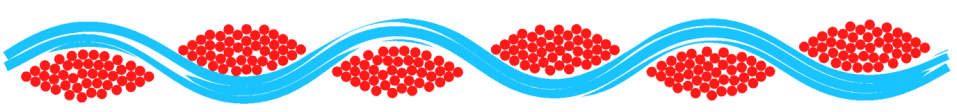

Fig. 2 Cuts of configuration before weaving (a), and of computed configuration for a plain weave (b) and a twill weave (c) patches
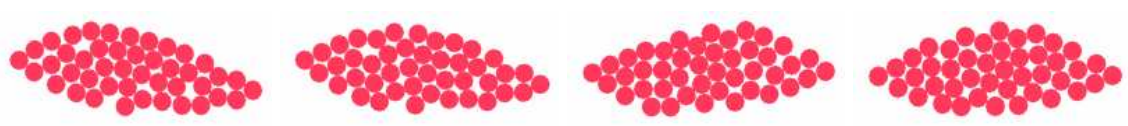

Fig. 3 Variation of cross-section shapes along a warp yarn for twill weave 


\subsection{Application of loading tests}

Once the initial configuration has been computed, various loading cases can be simulated by applying appropriate loadings (displacements or forces) to rigid bodies attached to each side of the patches. Biaxial tension loadings are applied up to a $2 \%$ extension in the warp direction, with a ratio $\alpha$, taken successively equal to 0 and 1 , for the extension in the weft direction. Typical J-shape force/displacement curves are obtained (Fig. П). Nonlinear effects at the start of the curves are related to the compaction of cross-sections as the tensile force increases.
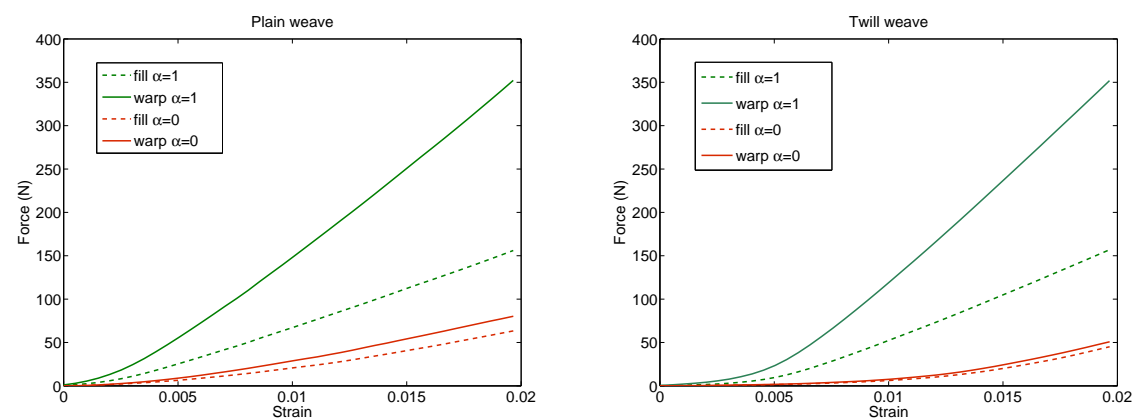

Fig. 4 Force/strain curves for biaxial tension tests for plain weave and twill weave

\section{Strain measures at the scale of yarns}

The formulation of mesoscopic models to represent the behaviour of yarns requires to define measures for both strains and stresses at the scale of yarn. The definition of a stress measure is difficult because stresses within yarns are of two different kinds : continuous stresses inside yarns, and discrete contact-friction interactions between fibers.

As a first step, the definition of a strain measure at the scale of yarn is easier. To approximate such a quantity, we propose to consider each yarn as a continuum, and to compute, as post-processing, Green-Lagrange strain tensors using a 3D finite element mesh based on the nodes defined on fibers (Fig. 5). Once the mesh connectivity has been defined for the initial configuration, nodal displacements and strain tensors can be easily derived. It is also possible to consider strains generated by displacements only between two given loading increments.

The plotting of horizontal strains due to the initial forming (Fig. 6) shows strong inhomogeneities. Zones looking like diagonal shear bands can be observed on the cuts of strains in both horizontal and vertical directions (Fig. 7 and 8). 

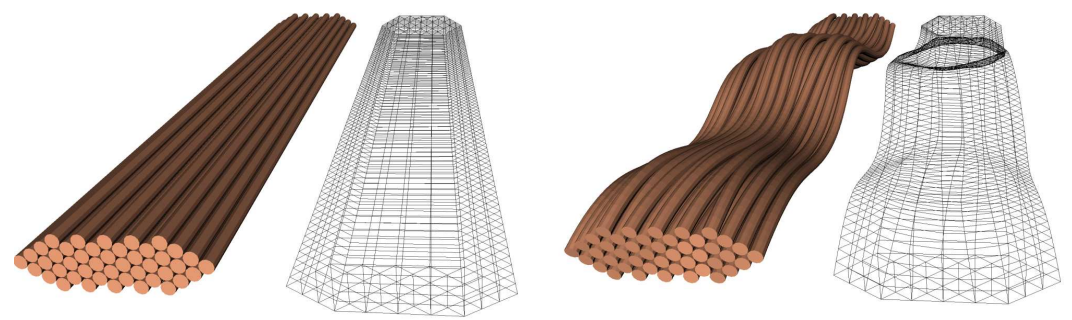

Fig. 5 Fibers of a yarn, and corresponding 3D meshes in two different configurations

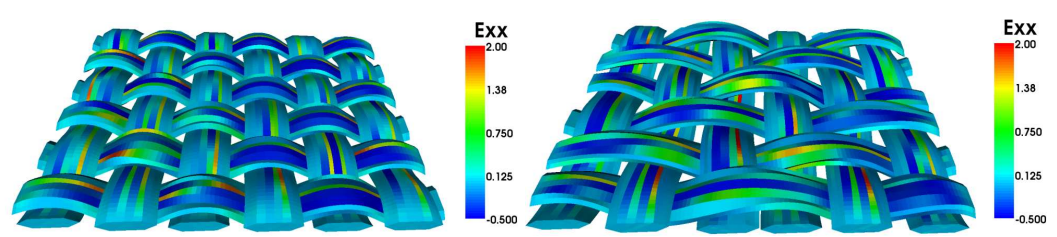

Fig. 6 Horizontal strains in yarns generated by the simulation of the weaving process

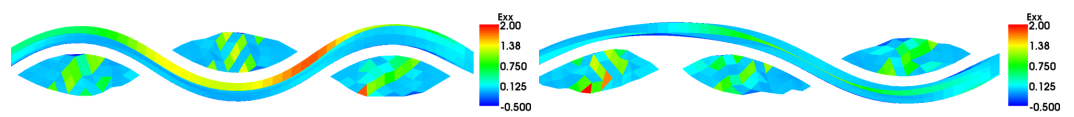

Fig. 7 Cuts of horizontal strains in yarns generated by the simulation of the weaving process

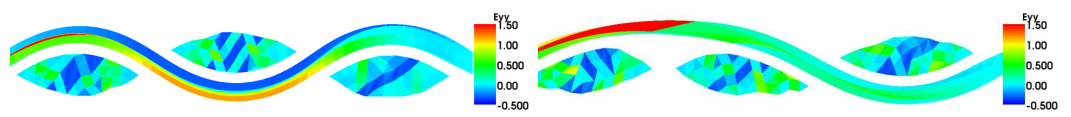

Fig. 8 Cuts of vertical strains in yarns generated by the simulation of the weaving process

Similar inhomogeneities can be observed for strains induced by the equibiaxial tension (Fig. D). In this case, the axial strain is twice higher on the sides of the yarn than in the center. The appearance of shear bands shows a rearrangement of fibers in the center of the yarn. This rearrangement reduces the heigth of the cross-section, allowing fibers to undergo lower extensions.

Inhomogeneities revealed by this post-processing are of first importance since they raise the question of using continuous models to describe the behaviour of yarns.

\section{Conclusion}

Models and algorithms developped for the simulation of the mechanical behaviour of entangled media can be applied for the modelling of woven structures. Patches of fabric made of few hundreds of fibers can be considered by the model. The simula- 

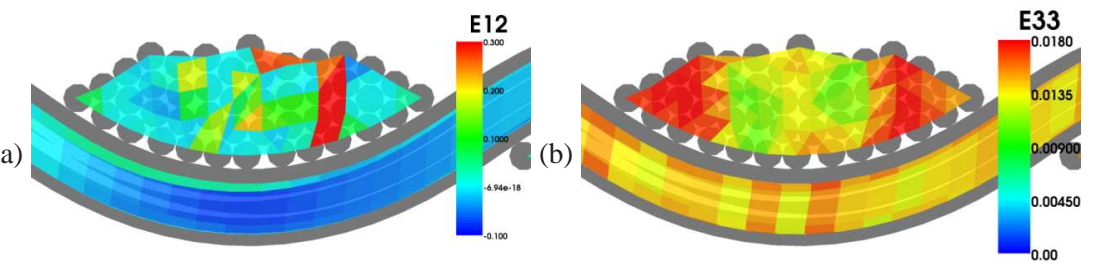

Fig. 9 Shear strains (a) and axial strains (b) in yarns generated by equibiaxial tension

tion of the weaving process provide accurate geometrical descriptions of both yarns trajectories and varying shapes of yarns cross-sections. The mechanical response of the fabric can then be characterized by simulating typical loading tests. The computation of 3D strains at the scale of yarns reveals strong inhomogeneities and raises the question of the validity of considering yarns as continuums.

\section{References}

1. Boisse, P., Zouari, B., Gasser, A.: A mesoscopic approach for the simulation of woven fibre composite forming. Composites Science and Technology 65(3-4), 429-436 (2005)

2. Durville, D.: Numerical simulation of entangled materials mechanical properties. Journal of Materials Science 40(22), 5941-5948 (2005)

3. Durville, D.: Recent Advances in Textile Membranes and Inflatable Structures, chap. Finite element simulation of the mechanical behaviour of textile composites at the mesoscopic scale of individual fibers, pp. 15-34. Springer (2008)

4. Finckh, H.: Numerical simulation of mechanical properties of fabrics - weaving / numerische simulation der mechanischen eigenschaften textiler flächengebilde - gewebeherstellung. Proceedings of the German 3rd LS-DYNA Forum 2004, Bamberg, Germany (2004)

5. Lomov, S., Ivanov, D., Verpoest, I., Zako, M., Kurashiki, T., Nakai, H., Hirosawa, S.: Meso-fe modelling of textile composites : Road-map, data flow and algorithms. Composites Science and Technology 67, 1870-1891 (2007)

6. Verpoest, I., Lomov, S.: Virtual textile composites software wisetex : Integration with micromechanical, permeability and structural analysis. Composites Science and Technology 65, 2563-2574 (2005) 